\title{
Reduction in Wnt9b and associated gene expression in the embryonic midface of CLIFr mice with heritable cleft lip and palate
}

\author{
Brennan Takagi ${ }^{1}$, Trudy Hong ${ }^{1}$, Thomas E. Hynd ${ }^{1}$, Keith S. K. Fong ${ }^{1}$, Ben Fogelgren ${ }^{1}$, \\ Kazuaki Nonaka ${ }^{2}$, Scott Lozanoff ${ }^{1}$ \\ ${ }^{1}$ Department of Anatomy, Biochemistry and Physiology, University of Hawaii Fobn A. Burns School of Medicine, Honolulu, Hawaii, USA \\ ${ }^{2}$ Section of Pediatric Dentistry, Faculty of Dentistry, Kyushu University, Fukuoka, Fapan
}

\begin{abstract}
Objectives: The CL/Fr mouse displays cleft lip and palate (CLP) at a rate of 35\%. The clf1 mutation is associated with CLP in related " $\mathrm{A}$ " strain mice and affects the gene Wnt9b. The purpose of this study was to determine tissue specific expression of Wnt9b during facial prominence morphogenesis in CL/Fr mice and provide new details concerning gene variants associated with CLP.

Methods: Facial prominences from CLP(-) and CLP(+) CL/Fr and 3H1 wild-type (WT) mice at embryonic day 11.5 (E11.5) were collected for expression assays (DNA microarray analysis, GRT-PCR, immunostaining, and in situ hybridization). A modified Chi square test was used to analyze microarray data while a student t-test was used to statistically compare qRT-PCR values $(p<0.05)$.

Results: There was a partial and variable loss of Wnt9b in facial prominences of E11.5 CLP susceptible CL/Fr mice, with a greater loss associated with CLP(+). Two genes in the clf2 locus, Adcy2 and Ube2q11 also showed decreased expression. Two regulators of palatogenesis, Runx2 and Osr2 were significantly downregulated, while an inhibitor of cell proliferation, somatostatin (Sst), was elevated in $\mathrm{CLP}(+)$ relative to $\mathrm{CLP}(-)$ mice.

Conclusion: Results indicate a role for Wnt9b in the pathogenesis of CLP and supports previous reports concerning its involvement with CLP in "A" strain mice. Misexpression of Sst suggests that it may be a downstream target of Wnt9b causing reduced overall growth possibly hindering fusion of facial prominences and contributing to the development of CLP.
\end{abstract}

Keywords: cleft lip and palate; CL/Fr mice; craniofacial; Wnt9b

Anatomy 2016;10(3):182-192 @2016 Turkish Society of Anatomy and Clinical Anatomy (TSACA)

\section{Introduction}

Orofacial clefts (cleft lip with or without cleft palate) are one of the most common birth defects. It occurs about 1 in 1000 live births in the United States, and over 7,000 new cases are expected annually with estimated lifetime treatment costs amounting to over $\$ 697$ million nationally. ${ }^{[1,2]}$ Orofacial malformations are debilitating since they can lead to difficulties in breathing, suckling, facial expression and speech in the child, and can also cause emotional hardships for the associated family. Both genetic and environmental factors contribute to these malformations and identifying the mechanisms involved will help to provide information regarding prenatal screening methods and treatment therapies in humans. ${ }^{[3]}$

The critical stage that is affected in CLP involves the formation of the upper lip and palate, which is normally completed when the laterally projecting maxillary prominences $(\mathrm{MxP})$ and lateral nasal prominences (LNP), fuse with the medial nasal prominences (MNP). This process is regulated by complex genetic signaling pathways, and relies on precise timing of the outgrowth of the prominences, contact between epithelia of the prominences in the nasal fin region, and eventual disintegration of contiguous tissue borders to form the upper lip. This event typically occurs at 41 days of gestation in 
humans and embryonic day 11.5 (E11.5) in mice. Interruption of any component of this process leads to failure of fusion and CLP.

Similarities in the genetic pathways involved in craniofacial morphogenesis in mice and humans, allow for the use of mouse models to study and understand the pathogenesis of CLP in humans. The "A" strain family of inbred mice has been useful because it exhibits spontaneous CLP ${ }^{[4,5]}$ Of the "A" strains, CL/Fr, created by an outcross of the A/J strain with selection for CLP, has the highest risk of CLP, occurring in $35 \%$ of newborns. ${ }^{[5,6]}$ Affected newborns have altered facial geometry and abnormal morphological features that are similar to those seen in humans with CLP.

Two disease loci, clf1 and clf2, are involved in the "A" strain defect. ${ }^{[7-10]}$ The clf1 locus on distal chromosome 11 contains the genes $W n t 9 b$ and $W n t 3 .^{[7,9]}$ This region is syntenic to the human chromosome $17 \mathrm{q} 21$, which has been associated with nonsyndromic CLP in humans. ${ }^{[1,12]}$ Furthermore, SNPs within the WNT9B gene have been associated with nonsyndromic CLP in a Brazilian population. ${ }^{[13]} W n t$ genes are expressed strongly during mouse craniofacial development and are involved in cell-to-cell communication, regulation of cell proliferation, and patterning in many developmental contexts. ${ }^{[14]}$ Previous gene-targeting analyses suggest that $W n t 9 b$ is the mutated gene involved in the clf1 region, contributing to CLP formation in $\mathrm{A} / \mathrm{WySn}$ mice. ${ }^{[15]} \mathrm{In}$ addition, it has been hypothesized that the presence of an intracisternal A particle (IAP) transposon near the $W n t 9 b$ gene at the $c l f 1$ locus and epistatic interaction of the $\operatorname{clf} 2$ locus leading to hypomorphic $W n t 9 b$ expression, may have a role in the development of CLP in these mice as well. ${ }^{[10,15,16]}$ However, it is still unclear how reduced levels of $W n t 9 b$ result in a cleft lip phenotype.

In this study we measured tissue specific expression and examined spatial expression patterns of $W n t 9 b$ during nasal fin development and fusion in CL/Fr mice to determine whether aberrant and hypomorphic expression of $W n t 9 b$ is associated with an elevated risk for CLP. We also offer new details on candidate genes that may be involved in CLP formation in CL/Fr mice.

\section{Materials and Methods}

\section{Animals}

All procedures were carried out in accordance with Institutional Animal Care and Use Committee (IACUC) specifications and performed following protocols approved by the University of Hawaii Laboratory of Animal Services. Adult mice were housed under standard conditions with 12-hour light cycles and supplied with tap water and Rodent Mouse Chow ad libitum. Breeding pairs were re-derived from an existing colony and reciprocally crossed. Females were examined for a vaginal plug at 8:00 $\mathrm{AM}$ and if absent, they were removed and re-mated the next day. The day on which a vaginal plug was observed was designated as day 0.5 of gestation (E0.5). Embryo collection was also performed on adult females from crosses between $3 \mathrm{H} 1$ mice. These embryos served as a control (WT) due to an extremely low incidence of CLP $(<1 \%)$ in the $3 \mathrm{H} 1$ strain.

\section{Tissue collection}

Pregnant CL/Fr females were isofluorane-anesthetized and sacrificed by cervical dislocation at 11.5 days of gestation. Embryos were obtained via caesarian section, placed in ice-cold phosphate-buffered saline (PBS) (pH 7.4) and verified to be Theiler stage 19 (E11.5). Facial prominences were isolated from the underlying telencephalon and the right half of the face was separated from the left along the median plane between the MNPs. Each half of the face contained one MNP, one LNP, and one MxP. The halves were placed in individual tubes containing 400 $\mu$ of RNAlater (Ambion, Thermo Fisher Scientific, North Ride, NSW, Australia) and stored at $4^{\circ} \mathrm{C}$ for 24 hours. The tissues were then removed from the RNAlater for processing. WT tissues were obtained from embryos derived from $3 \mathrm{H} 1+/+$ matings and collected in a similar fashion. Initial observance of phenotype was scored for non-cleft (normal) or bilateral cleft.

\section{Quantitative real time-PCR}

Total RNA from CL/Fr and 3H1 facial prominences was purified using the RNeasy Mini Kit according to the manufacturer's protocol (Qiagen, Valencia, CA, USA). cDNA was synthesized from $400 \mathrm{ng}$ of each RNA sample using the iScript cDNA Synthesis Kit (Bio-Rad Laboratories, Hercules, CA, USA). All procedures were performed according to the manufacturer's protocols. Gene expression was determined by quantitative real-time PCR (qRTPCR) in triple replicates ( $25 \mu \mathrm{L}$ final volume) using the $\mathrm{iQ}$ SYBR Green Supermix reaction procedure (Bio-Rad Laboratories) with the CFX96 Real-Time Detection System and C1000 thermocycler (Bio-Rad Laboratories). PCR conditions consisted of an initial 2-min denaturation at $94^{\circ} \mathrm{C}$, followed by 40 reactions cycled through denaturation for $15 \mathrm{sec}$ at $94^{\circ} \mathrm{C}$, annealing for $30 \mathrm{sec}$ (temperature dependent on primer pair; see Table 1), and extension for $60 \mathrm{sec}$ at $72^{\circ} \mathrm{C}$. The threshold cycle was established at the linear portion of the $\log$ scale curve and expression levels were normalized to Gapdh or Actb and calculated using the $2^{-\Delta \Delta^{C(t)}}$ method. ${ }^{[17]}$ Primer sets to amplify $W n t 9 b, W n t 3$, Trp63, Gapdh, Sst and Actb were optimized for SYBR green 
Table 1

PCR primers. Oligonucleotide sequences and specifications of primers used for quantitative RT-PCR. Sense oligonucleotides (F) and antisense (R) are listed.

\begin{tabular}{|c|c|c|c|c|}
\hline Primer & 5'-Sequence-3' & $\begin{array}{c}\text { Melting } \\
\text { temperature }\end{array}$ & $\begin{array}{c}\text { Annealing } \\
\text { temperature }\end{array}$ & Efficiency \\
\hline Wnt9b F & GCT GGG AAG ATC TIT GAT GG & $53.4^{\circ} \mathrm{C}$ & $59^{\circ} \mathrm{C}$ & $99.8 \%$ \\
\hline Wnt9b R & TCC ATT CTT GCC TTG TAC CC & $54.8^{\circ} \mathrm{C}$ & & \\
\hline Wnt3 F & AGT TCC TTG TGG GCT CCT GT & $61.1^{\circ} \mathrm{C}$ & $60^{\circ} \mathrm{C}$ & $94.4 \%$ \\
\hline Wnt3 R & CCT CGG TGT CTG CTG GTT AG & $60.8^{\circ} \mathrm{C}$ & & \\
\hline$\beta$-actin $F$ & CAT CCG TAA AGA CCT CTA TGC CAA C & $57.3^{\circ} \mathrm{C}$ & $52{ }^{\circ} \mathrm{C}$ & $95.7 \%$ \\
\hline$\beta$-actin R & ATG GAG CCA CCG ATC CAC A & $59.0^{\circ} \mathrm{C}$ & & \\
\hline Trp63 F & CAT AGC ATG AGC TGA ACC AC & $53.6^{\circ} \mathrm{C}$ & $51^{\circ} \mathrm{C}$ & $97.5 \%$ \\
\hline Trp63 R & GCT TTC CCA AGG TAT GAA AC & $51.5^{\circ} \mathrm{C}$ & & \\
\hline Gapdh F & GCA TCT TGG GCT ACA CTG AG & $55.6^{\circ} \mathrm{C}$ & $59^{\circ} \mathrm{C}$ & $100 \%$ \\
\hline Gapdh R & GGT GGT CCA GGG TTT CTT AC & $55.3^{\circ} \mathrm{C}$ & & \\
\hline Sst $F$ & AGA GGT CTG CCA ACT CGA AC & $56.9^{\circ} \mathrm{C}$ & $53^{\circ} \mathrm{C}$ & $102.5 \%$ \\
\hline Sst $R$ & GGC CAG GAG TTA AGG AAG AG & $54.8^{\circ} \mathrm{C}$ & & \\
\hline
\end{tabular}

real-time detection (see Table 1 for description of primer sets). As per the Minimum Information for Publication of Quantitative Real-Time PCR Experiments (MIQE) guidelines, primers were tested for specificity and PCR efficiencies were within acceptable values (range: $94.4-100 \%){ }^{[18]}$ For the initial qRT-PCR analysis, samples sizes consisted of $18 \mathrm{WT}, 15 \mathrm{CLP}(-)$ and $8 \mathrm{CLP}(+)$.

\section{In situ hybridization}

A total of 12 embryos from 3 litters, four $3 \mathrm{H} 1$ wild-type, four CL/Fr non-cleft, and four CL/Fr cleft embryos, were collected at E11.5, fixed in 4\% PFA and stored in 100\% methanol at $-20^{\circ} \mathrm{C}$. The embryos were subjected to in situ hybridization to detect $W n t 9 b$ expression. A $4.3 \mathrm{~kb}$ fragment corresponding to the $W n t 9 b \mathrm{mRNA}$ was cloned into a pSPORT-P plasmid vector flanked by T7 and Sp6 transcriptional promoters. ${ }^{[19]}$ The plasmid was amplified by transforming bacteria, purified by column filtration (Sigma-Aldrich, St. Louis, MO, USA) and linearized with NotI (New England Biolabs, Ipswich, MA, USA) to generate antisense and SalI (New England Biolabs) sense ribonucleotide probes. The RNA probe was synthesized using DIG RNA labeling mix (Roche Diagnostics, Indianapolis, IN) and Sp6 and T7 RNA Polymerase (Promega, Madison, WI, USA) based on manufacturer's specifications. For in situ hybridization, embryos in methanol were rehydrated, hybridized with riboprobe, and incubated with anti-DIGAP Fab (Roche) as previously described..$^{[20]}$ DIG-labeled RNAs were detected with NBT/BCIP (Roche), cleared with glycerol, and examined for staining in the developing craniofacial region (MNPs, LNPs, and MxPs).

\section{Immunohistochemistry}

3H1 WT embryos, CLP(+) embryos, and CLP(-) cleft embryos were harvested at E11.5, rinsed in ice cold PBS, and flash frozen in optimum cutting temperature (OCT) compound. Sagittal and transverse sections $(10 \mu \mathrm{m})$ were cut with selection for the contact point between facial prominences and transferred to polylysine coated microscope slides (Thermo Fisher Scientific, Waltham, MA, USA). Slides were allowed to dry for up to one hour at room temperature until fixed in ice cold methanol for 20 mins at $-20^{\circ} \mathrm{C}$. Slides were blocked with $5 \%$ normal donkey serum in PBS for 30 mins and incubated overnight in goat anti-Wnt $9 b$ primary antibody $(1: 100 ; \mathrm{S} \& \mathrm{D}$ Bioscience, Manassas, VA, USA). Samples were incubated with DyLight donkey $\alpha$-goat secondary antibody. To prevent rapid loss of fluorescence and photobleaching during microscopic examination, Vectashield Mounting Media for Fluorescence with DAPI $(1.5 \mu \mathrm{g} / \mathrm{ml}$, Vector Laboratories, Burlingame, CA, USA) was used for tissue section mounting. Identical exposure length and image processing was achieved by collecting tissue images at the constant magnifications to obviate unparalleled changes in fluorescence during processing.

\section{DNA microarray analysis}

Three biological replicates were obtained for control, CLP(-) and test CLP(+) samples. Two sets of tissue comparisons were undertaken based on these samples. First, age-matched individual CLP(-) control samples $(\mathrm{C} 1,2,3)$ were compared to individual $\mathrm{CLP}(+)$ test samples $(\mathrm{T} 1,2,3)$. A second comparison was undertaken comparing 
pooled CLP(-) samples (P1) to corresponding CLP(+) samples (P2). Expression profiles were generated for these comparisons.

The anatomical right halves of the faces collected were used for the array and the anatomical left halves were reserved for corresponding qRT-PCR analysis. Total RNA was purified using the NucleoSpin RNA XS Total RNA isolation kit according to the manufacturer's protocol (Macherey-Nagel, Bethlehem, PA, USA). Gene expression profiling was achieved using the whole mouse genome 4×44K microarray kit (Agilent Technologies, Santa Clara, CA, USA). Data was analyzed using the GeneSpring Analysis Platform (Agilent Technologies) to determine the number of genes expressed in the dataset.

Attention was directed to genes in the clf1 and clf2 loci, other CLP candidate genes and genes that have been found to regulate craniofacial morphogenesis. In order to better define significant expression changes of these and other candidate genes, a rigorous analysis, focusing on consistency between comparisons in directionality and degree of fold change, was developed and implemented. The analysis consisted of determining fold change that was calculated iteratively among the samples paired into all 16 possible comparisons. A short subroutine was written to identify positive or negative fold change above 1.5 for all 16 comparisons. A Chi-square statistical analysis was assessed for the number of comparisons in agreement. ${ }^{[2]]}$ Thus, we determined the number of comparisons that were in agreement with respect to a fold change that extended above or below the 1.5 -fold cut-off. This rigorous analysis applied a stringent level of statistical analysis to the array and enabled filtering of average gene expression comparisons as well as facilitating the identification of potential outliers.

\section{Results}

\section{Reduced expression of Wnt9b, but no significant difference in levels of Wnt3 and Trp63 RNA in the facial prominences of CL/Fr mice}

To determine whether $W n t 9 b$ expression is affected in $\mathrm{CL} / \mathrm{Fr}$ mice, as suggested by previous genetic studies, qRT-PCR was performed using E11.5 facial prominences from CLP(-), CLP(+) and WT samples (Table 1; Figure 1). On average, $W n t 9 b$ expression was reduced by $30 \%$ in the CLP(-) samples relative to WT mice (Figure 1a). Additionally, CLP $(+)$ samples showed an overall $50 \%$ decrease in expression of $W n t 9 b$ relative to $\operatorname{CLP}(-)$ embryos (Figure 1b). Since previous studies on $\mathrm{Pbx}$ compound mutants showed reduced expression of $W n t 3$ and $T r p 63$ in conjunction with reduced $W n t 9 b$, we also measured expression of these genes. ${ }^{[2]}$ No significant difference in the level of $W n t 3$ and $\operatorname{Trp} 63$ RNA was seen in the CLP(+) relative to CLP(-) embryos (Figure 1b).

To determine the spatial expression patterns of $W n t 9 b$ in the developing regions of the upper lip and palate, E11.5 WT, CLP(+) and CLP(-), embryos were subjected to whole mount in situ hybridization (Figure 2). Prominent $W n t 9 b$ staining can be seen in the distal ecto-
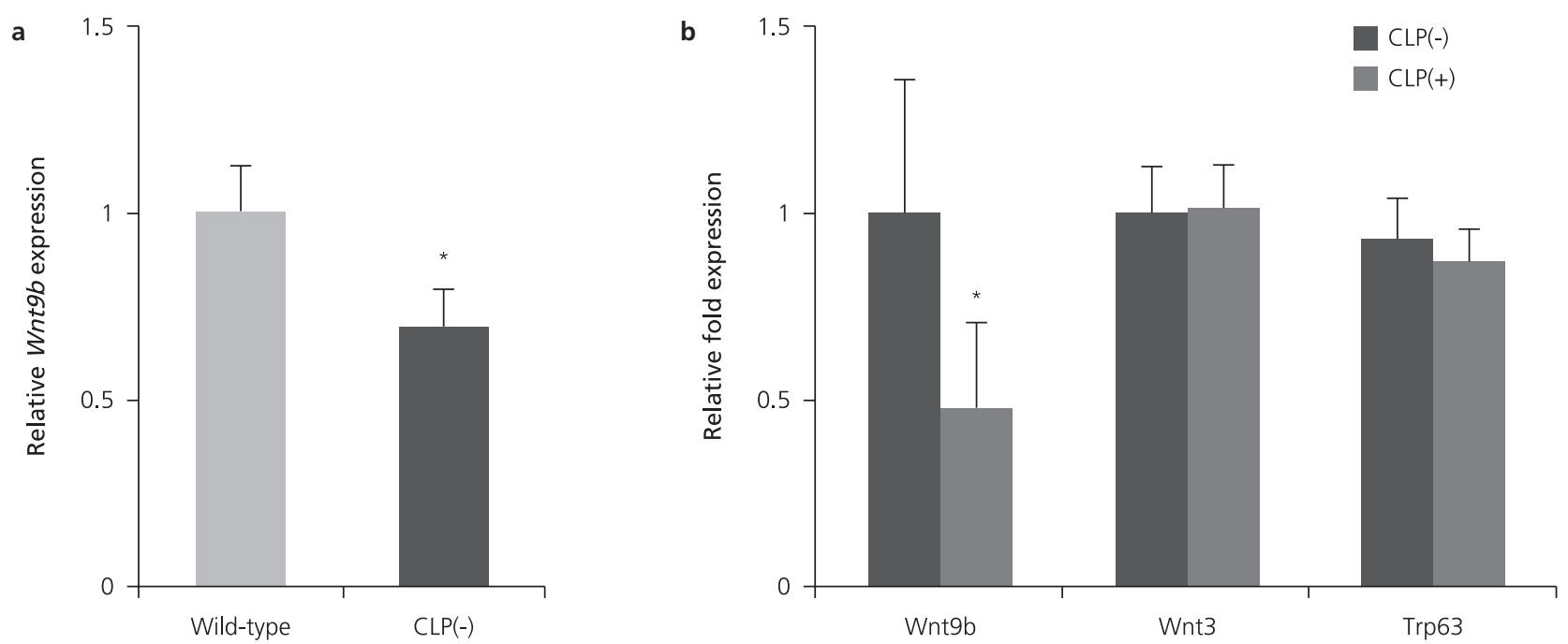

Figure 1. Reduced Wnt9b but not Wnt3 or Trp63 in CL/Fr embryos. The E11.5 facial prominences showing (a) reduced expression of Wnt9b in the $\mathrm{CLP}(-)$ relative to $3 \mathrm{H} 1$ wild-type mice as well as (b) CLP(+) relative to CLP(-) CL/Fr mice. However, no difference was seen for Wnt3 and Trp63 (B) in $\mathrm{CL} / \mathrm{Fr}$ mouse comparisons based on qRT-PCR analysis. $\mathrm{C}(\mathrm{t})$ was established at the linear portion of the log scale curve and ratio of Wnt9b to Actb was calculated using the $2^{-\Delta \Lambda C(t)}$ method. ${ }^{*} p<0.01$. 

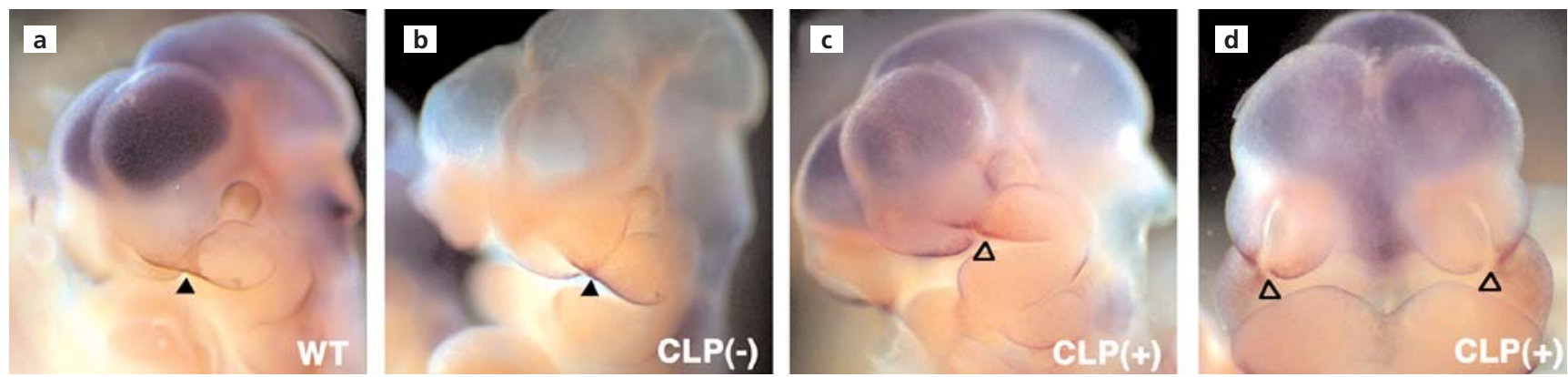

Figure 2. In situ hybridizations for Wnt9b in whole-mount E11.5 embryos. (a) 3H1 WT, (b) CLP(-) CL/Fr, (c, d) CLP(+) CL/Fr. Wnt9b is detected in the distal ectoderm of the MNP, LNP and MxP and in the epithelial contact sites between the fusing prominences. The CLP(+) CL/Fr embryos (c and $\mathbf{d})$ show slightly reduced Wnt9b expression in these areas relative to the WT and CLP(-) CL/Fr (a and $\mathbf{b})$. Solid triangles represent contact of the prominences in WT and CLP(-) mice. Open triangles indicate lack of contact in the CLP(+). CLP(+): presence of cleft lip and palate; CLP(-): absence of cleft lip and palate. [Color figure can be viewed in the online issue, which is available at www.anatomy.org.tr]

derm of the MNP, LNP, and MxP of the fusing prominences of WT and CLP(-) embryos. The CLP(+) embryos show reduced $W n t 9 b$ expression in these areas relative to the CLP (-) and WT embryos, consistent with our qRT-PCR results (Figures $\mathbf{1}$ and 2).

To visualize the localization of $W n t 9 b$ protein in the embryonic facial prominences during the critical time of nasal fin fusion, IHC was performed on a total of nine E11.5 WT, CLP(-) and CLP(+) mice (Figure 3). Wnt9b fluorescence is prominent in the area of the distal epithelial cells lining the facial prominences in WT mice (Figure 3a). $W n t 9 b$ fluorescence is also seen in the epithelial cells lining the mandibular component of the first branchial arch. These results are consistent with previous reports by Lan et al. (2006). Sections from CLP(-) mice display presence of $W n t 9 b$ protein (Figure $3 \mathbf{c}$ and $\mathbf{d}$ ); however, the staining is not quite as robust compared to the WT facial prominences. The staining pattern in CLP(+) facial prominences is fundamentally different compared to both WT and $\mathrm{CLP}(-)$ specimens. $\mathrm{CLP}(+)$ prominences show greatly reduced $W n t 9 b$ expression (Figure $3 \mathbf{b}$ ). The expression also appears to occur as a punctate pattern within the
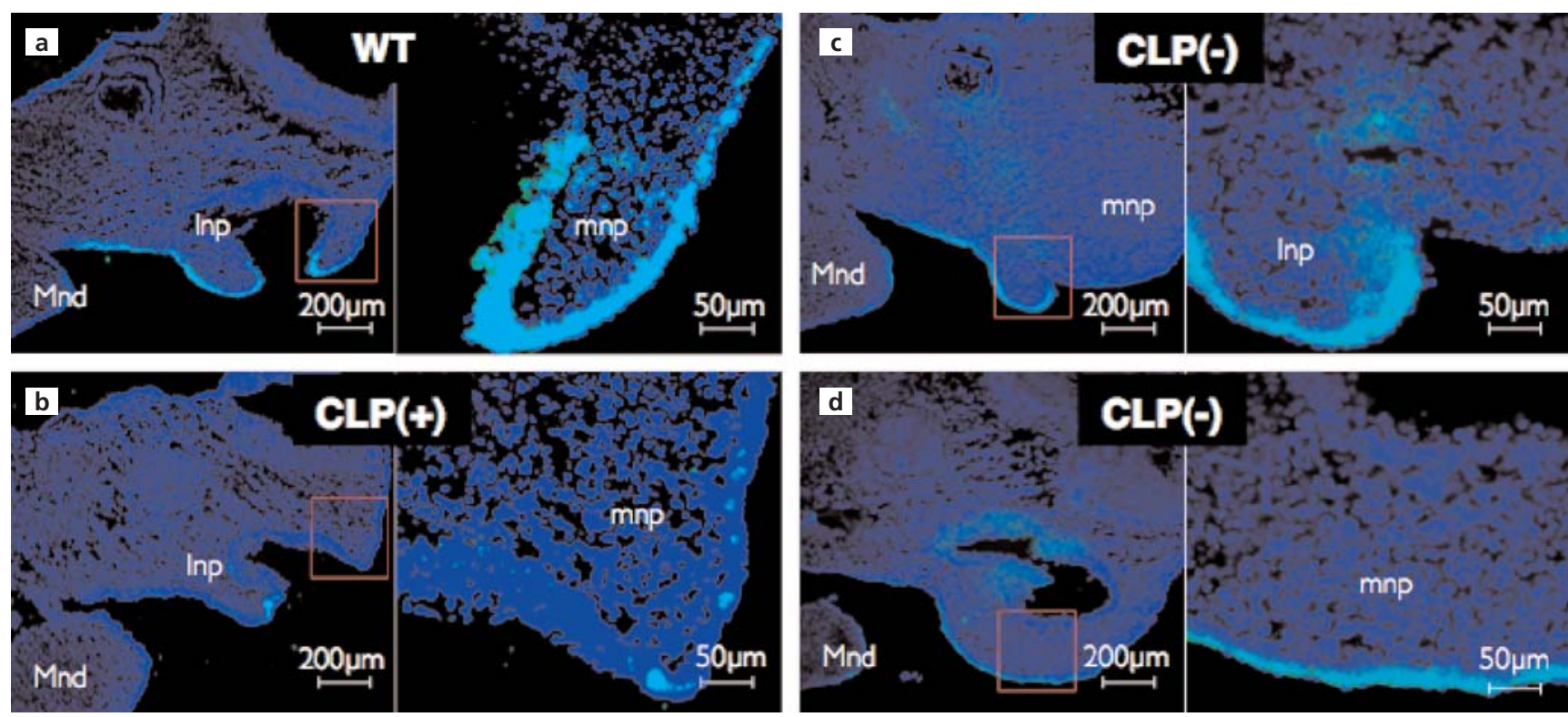

Figure 3. Immunofluorescence of Wnt9b in E11.5 3H1 WT, CLP(-) and CLP(+) CL/Fr embryos. Strong localization of Wnt9b (light blue) is seen in the facial prominence epithelial cells of E11.5 WT (a) and CLP(-) CL/Fr (c and $\mathbf{d}$ ) embryos, relative to negative control (no primary antibody; data not shown). Reduced Wnt9b is seen in the epithelial cells of CLP(+) CL/Fr (b) samples compared to CLP(-) CL/Fr and WT samples. The CLP(+) is characterized by a punctate pattern of Wnt9b expression. Areas indicated by the red boxes were $4 \times$ magnified and are shown in the right panels. [Color figure can be viewed in the online issue, which is available at www.anatomy.org.tr] 
epithelial rather than a continuous band of staining in WT and $\mathrm{CLP}(-)$ specimens (Figure 3b). There also appears to be a dramatic reduction of $W n t 9 b$, in terms of the number of epithelial cells as well as the strength of fluorescence in the facial epithelium of the CLP(+) embryos.

\section{Differential gene expression in the faces of CL/Fr mice}

Microarray-based gene expression profiling was performed to identify genes that were differentially expressed in the facial prominences during fusion of the facial prominences in E11.5 CL/Fr mice with reduced levels of $W n t 9 b$ (Figure 4). Of 41,267 probes on the Agilent Mouse GE 4x44K microarray, more than 30,000 of these probes were expressed in the facial prominences in at least one sample. Of these detected probes, 117 had at least a 2fold change in expression level with a significant probability $(\mathrm{p}<0.05$, Student's t-test). After the removal of probes corresponding to unknown genes and genes on sex chromosomes (gender segregation was not performed), 48 genes were identified as having increased expression in the CLP(+) samples (Table 2) compared with 46 exhibiting decreased expression (Table 3).

Consistent with aforementioned expression assays, $W n t 9 b$ but not $W n t 3$ or $T r p 63$ expression was found to be significantly reduced in the gene array analysis of CLP(+) tissue ( -3.11 fold; $\mathrm{p}<0.01,-1.50$ fold; $\mathrm{p}<0.02$, and -1.03 fold; $\mathrm{p}<0.55$ respectively). Under rigorous analysis, $W n t 9 b$ differed between all possible comparisons except one (Rigorous Analysis, 15/16, Table 3). Two genes within the clf 2 locus showed significant decreases based on the microarray analysis included adenylate cyclase $(A d c y 2)$ $(-2.22$ fold; $\mathrm{p}<0.05)$ and ubiquitin-conjugating enzyme E2Q family like 1 (Ube2q11), (-1.83 fold; p <0.01) (Table 3).

The greatest change in expression based on our microarray analysis was for somatostatin (Sst) (Table 2). Expression was significantly changed in CLP(+) tissue (13.35-fold; $\mathrm{p}<0.05$; rigorous analysis: $\chi^{2}=6.25, \mathrm{p}<0.01$, d.f. $=1, \mathrm{~N}=16)$. A 1.72 -fold up-regulation of $s s t(\mathrm{p}<0.01)$ was detected among individual CLP(+) tissues by qRTPCR (Figure 5). Although this increase was not as dramatic as indicated from our array data, it supports the microarray analysis since it falls within the large range of positive fold changes for Sst expression in cleft samples

\section{Discussion}

The disruption of canonical WNT/ $\beta$-catenin signaling pathway has been implicated in the pathogenesis of CLP ${ }^{[2,2,24]}$ In our study, we have demonstrated that the development of clefts in CL/Fr mice is associated with reduced expression of $W n t 9 b$, an important member of this

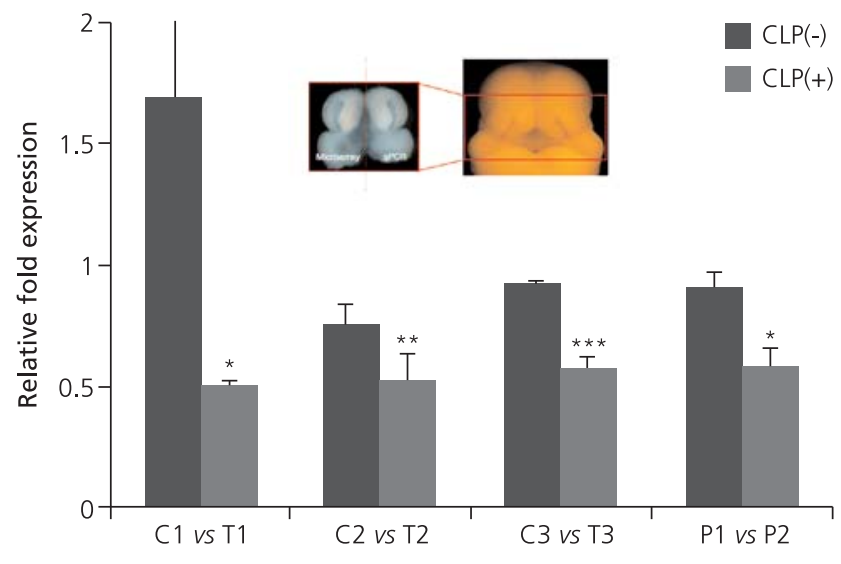

Figure 4. Relative Wnt9b expression in the face of $C L P(-)$ and $C L P(+)$ E11.5 embryos used for the microarray. Corresponding facial prominences were utilized for both qRT-PCR and microarray analyses (inset). Relative fold comparison derived from qRT-PCR analysis showing reduced Wnt9b expression in individual $C L P(+)$ embryos $(T 1,2,3)$ compared with corresponding individual $\mathrm{CLP}(-)$ samples $(\mathrm{C} 1,2,3)$. Pooled samples from $\mathrm{CLP}(+)$ animals (P1) compared to CLP(-) embryos (P2) show consistent results. Reduced expression of Wnt9b in the CLP(+) samples is consistent with the microarray data (see Table 3); $: p<0.01,{ }^{* *}: p<0.05,{ }^{* *}: p<0.001$.

signaling pathway and CLP candidate gene. $W n t 9 b$ was the seventh most significantly down-regulated gene in our microarray analysis with greater than a 3 -fold reduction in CLP(+) compared to CLP(-) CL/Fr embryos (Table 3). This result was confirmed by qRT-PCRs on facial prominences corresponding to those used in the array (Figures 1 and 4). This difference is greater compared to cleft versus non-cleftA/WySn mice where transcription of $W n t 9 b$

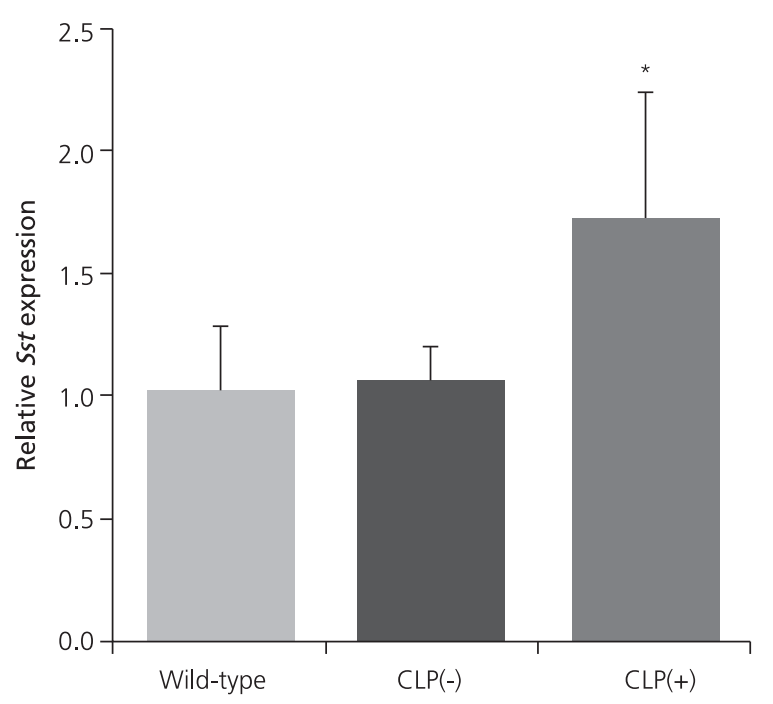

Figure 5. Expression of Sst in CL/Fr facial prominences. Increased expression of Sst in $\mathrm{CLP}(+)$ tissues compared to $3 \mathrm{H} 1$ wild-type and $\mathrm{CLP}(-) \mathrm{CL} / \mathrm{Fr}$ tissues based on qRT-PCR of E11.5 facial prominences. ${ }^{*} p<0.01$. 
appears to be affected by the methylated state of IAP. ${ }^{[16]}$ $W n t 9 b$ was also reduced in CLP(-) embryos compared to wild-type mice (Figure 1a); however, the CL/P(-) CL/Fr mice did not display clefts. This suggests that a threshold amount of $W n t 9 b$ is likely required for normal facial morphogenesis, and minor reductions in $W n t 9 b$ as seen in
CLP(-) CL/Fr is not sufficient for cleft development supporting long held views of threshold growth mechanisms associated with cleft formation. ${ }^{[25]}$

Whole mount in situ hybridizations on E11.5 embryos indicated that $W n t 9 b$ expression occurred in the facial prominences specifically in the distal ectoderm of the MNP,

Table 2

Genes exhibiting greatest increased expression in $\mathrm{CLP}(+)$ compared to $\mathrm{CLP}(-)$ samples.

\begin{tabular}{|c|c|c|c|c|}
\hline Gene symbol & p-value & Fold change & Rigorous analysis & Gene name \\
\hline Sst & 2.33E-02 & 13.35 & $13 / 16, p=0.01$ & somatostatin \\
\hline D730050B12Rik & 8.39E-03 & 7.42 & $16 / 16, p=6.33 E-05$ & RIKEN cDNA D730050B12 gene \\
\hline Mstn & $1.72 \mathrm{E}-02$ & 6.05 & $15 / 16, p=4.65 E-04$ & myostatin \\
\hline Kenj12 & 4.60E-02 & 5.45 & $12 / 16, p<0.05$ & potassium inwardly-rectifying channel, subfamily J, member 12 \\
\hline Ldhd & 4.80E-02 & 5.35 & $13 / 16, p=0.01$ & lactate dehydrogenase $\mathrm{D}$ \\
\hline Olfr1423 & 4.52E-02 & 5.14 & $12 / 13, p<0.05$ & olfactory receptor 1423 \\
\hline BC049349 & 4.17E-02 & 5.13 & $13 / 16, p=0.01$ & cDNA sequence BC049349 \\
\hline Olfr146 & $4.81 \mathrm{E}-02$ & 4.82 & $13 / 16, p=0.01$ & olfactory receptor 146 \\
\hline Gsbs & 3.23E-02 & 4.02 & $12 / 13, p<0.05$ & G substrate \\
\hline Ceacam2 & 3.79E-02 & 4.01 & $14 / 16, p=2.69 E-03$ & carcinoembryonic antigen-related cell adhesion molecule 2 \\
\hline LOC 100044500 & $5.24 \mathrm{E}-03$ & 3.86 & $14 / 16, p=2.69 E-03$ & similar to Dsg2 protein \\
\hline Taf4b & 3.10E-04 & 3.69 & $16 / 16, p=6.33 E-05$ & TAF4B RNA polymerase ॥, TATA box binding protein (TBP)-associated factor \\
\hline Gsbs & 2.17E-02 & 3.67 & $12 / 13, p<0.05$ & G substrate \\
\hline Vnn3 & 4.82E-02 & 3.66 & $13 / 16, p=0.01$ & vanin 3 \\
\hline$\| 5$ & $6.79 \mathrm{E}-03$ & 3.39 & $15 / 16, p=4.65 E-04$ & interleukin 5 \\
\hline Nefm & 2.71E-02 & 3.29 & $13 / 16, p=0.01$ & neurofilament, medium polypeptide \\
\hline Sncg & $2.58 \mathrm{E}-02$ & 3.14 & $13 / 16, p=0.01$ & synuclein, gamma \\
\hline Nefl & 4.46E-02 & 3.08 & $12 / 13, p<0.05$ & neurofilament, light polypeptide \\
\hline Ppp2r2C & 4.64E-02 & 3.02 & $12 / 13, p<0.05$ & protein phosphatase 2 (formerly $2 A$ ), regulatory subunit $B(P R 52$ ), gamma isoform \\
\hline $\mathrm{H} 28$ & 2.19E-02 & 2.97 & $13 / 16, p=0.01$ & histocompatibility 28 \\
\hline$\| l d r 2$ & 2.03E-02 & 2.83 & $14 / 16, p=2.69 E-03$ & immunoglobulin-like domain containing receptor 2 \\
\hline Lin28a & 3.27E-02 & 2.8 & $12 / 13, p<0.05$ & lin-28 homolog A (C. elegans) \\
\hline Rnf17 & $1.42 \mathrm{E}-02$ & 2.71 & $16 / 16, p=6.33 E-05$ & ring finger protein 17 \\
\hline Synm & 7.83E-03 & 2.65 & $12 / 13, p<0.05$ & synemin, intermediate filament protein \\
\hline Igdcc3 & $3.11 \mathrm{E}-02$ & 2.6 & $13 / 16, p=0.01$ & immunoglobulin superfamily, DCC subclass, member 3 \\
\hline Shroom3 & $3.82 \mathrm{E}-02$ & 2.47 & $13 / 16, p=0.01$ & shroom family member 3 \\
\hline Ttll6 & $1.96 \mathrm{E}-02$ & 2.47 & $14 / 16, p=2.69 E-03$ & tubulin tyrosine ligase-like family, member 6 \\
\hline Trim6 & 4.25E-02 & 2.46 & $13 / 16, p=0.01$ & tripartite motif-containing 6 \\
\hline Cartpt & $1.72 \mathrm{E}-02$ & 2.39 & $12 / 13, p<0.05$ & CART prepropeptide \\
\hline E330037M01Rik & $2.25 \mathrm{E}-03$ & 2.35 & $15 / 16, p=4.65 E-04$ & RIKEN CDNA E330037M01 gene \\
\hline Gm3560 & 8.29E-03 & 2.31 & $14 / 16, p=2.69 E-03$ & predicted gene 3560 \\
\hline Onecut1 & $3.21 \mathrm{E}-02$ & 2.31 & $13 / 16, p=0.01$ & one cut domain, family member 1 \\
\hline Accn4 & $1.46 \mathrm{E}-02$ & 2.29 & $14 / 16, p=2.69 E-03$ & amiloride-sensitive cation channel 4 , pituitary \\
\hline Rbpjl & 4.47E-02 & 2.27 & $<11$ of $16, p>0.05$ & recombination signal binding protein for immunoglobulin kappa J region-like \\
\hline E330037M01Rik & $1.61 \mathrm{E}-02$ & 2.22 & $13 / 16, p=0.01$ & RIKEN cDNA E330037M01 gene \\
\hline Onecut2 & $3.16 \mathrm{E}-02$ & 2.16 & $12 / 13, p<0.05$ & one cut domain, family member 2 \\
\hline Slc38a9 & 2.97E-02 & 2.13 & $12 / 13, p<0.05$ & solute carrier family 38, member 9 \\
\hline Cxcl13 & 8.62E-03 & 2.12 & $13 / 16, p=0.01$ & chemokine (C-X-C motif) ligand 13 \\
\hline Ntrk1 & 3.19E-02 & 2.12 & $12 / 13, p<0.05$ & neurotrophic tyrosine kinase, receptor, type 1 \\
\hline B130011D17Rik & $4.18 \mathrm{E}-02$ & 2.09 & $11 / 16, p=0.13$ & RIKEN CDNA B130011D17 gene \\
\hline Gsg1l & $3.76 \mathrm{E}-02$ & 2.07 & $11 / 16, p=0.13$ & GSG1-like \\
\hline Myh3 & $3.58 \mathrm{E}-02$ & 2.07 & $11 / 16, p=0.13$ & myosin, heavy polypeptide 3, skeletal muscle, embryonic \\
\hline Mreg & $1.37 \mathrm{E}-02$ & 2.04 & $13 / 16, p=0.01$ & melanoregulin \\
\hline Onecut2 & 4.19E-02 & 2.03 & $<11$ of $16, p>0.05$ & one cut domain, family member 2 \\
\hline Fam190a & $1.38 \mathrm{E}-02$ & 2.01 & $13 / 16, p=0.01$ & family with sequence similarity 190 , member $A$ \\
\hline
\end{tabular}


LNP, and MxP and at epithelial contact sites between the fusing prominences. However, expression was reduced in $\mathrm{CLP}(+)$ embryos (Figure 2). In addition to this observation, immunohistochemistry showed less $W n t 9 b$ in the epithelium of the facial prominences of $\operatorname{CLP}(+)$ embryos at a time when the facial prominences should fuse (Figure 3). As demonstrated in previous studies on $W n t 9 b-/-g e n e$ deletion generated mice, $W n t 9 b$ is essential for mesenchy- mal cell proliferation of facial prominences through WNT/ $\beta$-catenin signaling. ${ }^{[23]}$ Decreased $W n t 9 b$ expression in CL/Frmice likely leads to hypoplasia of the prominences and delayed contact and apoptosis at epithelial contact sites, failure of fusion, and CLP.

$W n t 9 b$, however, is likely not the only gene affected in $\mathrm{CL} / \mathrm{Fr}$ mice since CLP occurs in $35 \%$ of newborn CL/Fr mice, which is higher than the incidence seen in other " $\mathrm{A}$ "

Table 3

Genes exhibiting greatest decreased expression in the CLP(+) compared to CLP(-) samples (Note: splice variants included as separate entries).

\begin{tabular}{|c|c|c|c|c|}
\hline Gene symbol & p-value & Fold thange & Rigorous analysis & Gene name \\
\hline $\operatorname{Prl} 2 c_{3}$ & $3.14 \mathrm{E}-02$ & -4.93 & $14 / 16, p=2.69 E-03$ & prolactin family 2 , subfamily $c$, member 3 \\
\hline Pde1a & $2.06 \mathrm{E}-02$ & -3.85 & $14 / 16, p=2.69 E-03$ & phosphodiesterase $1 \mathrm{~A}$, calmodulin-dependent \\
\hline Calb2 & $1.37 \mathrm{E}-02$ & -3.64 & $13 / 16, p=0.01$ & calbindin 2 \\
\hline Pde1a & $1.89 \mathrm{E}-02$ & -3.50 & $13 / 16, p=0.01$ & phosphodiesterase $1 \mathrm{~A}$, calmodulin-dependent \\
\hline a & $3.68 \mathrm{E}-02$ & -3.35 & $13 / 16, p=0.01$ & nonagouti \\
\hline Cpxm2 & 4.85E-02 & -3.26 & $13 / 16, p=0.01$ & carboxypeptidase X 2 (M14 family) \\
\hline Calb2 & $1.20 \mathrm{E}-02$ & -3.21 & $13 / 16, p=0.01$ & calbindin 2 \\
\hline Gdf10 & $2.70 \mathrm{E}-02$ & -3.19 & $13 / 16, p=0.01$ & growth differentiation factor 10 \\
\hline Wnt9b & 4.05E-03 & -3.11 & $15 / 16, p=4.65 E-04$ & wingless-type MMTV integration site 9B \\
\hline Calb2 & $6.71 \mathrm{E}-03$ & -3.08 & $15 / 16, p=4.65 E-04$ & calbindin 2 \\
\hline Zfp572 & $2.11 \mathrm{E}-02$ & -3.05 & $14 / 16, p=2.69 E-03$ & zinc finger protein 572 \\
\hline Avpr1a & $6.10 \mathrm{E}-03$ & -3.02 & $14 / 16, p=2.69 E-03$ & arginine vasopressin receptor $1 \mathrm{~A}$ \\
\hline Orm3 & $4.62 \mathrm{E}-02$ & -2.99 & $12 / 16, p<0.05$ & orosomucoid 3 \\
\hline Penk & 2.07E-02 & -2.92 & $15 / 16, p=4.65 E-04$ & preproenkephalin \\
\hline Otop3 & $1.87 \mathrm{E}-02$ & -2.81 & $12 / 16, p<0.05$ & otopetrin 3 \\
\hline Pde1a & 4.04E-02 & -2.78 & $13 / 16, p=0.01$ & phosphodiesterase $1 \mathrm{~A}$, calmodulin-dependent \\
\hline Hdgfl1 & $1.44 \mathrm{E}-02$ & -2.76 & $14 / 16, p=2.69 E-03$ & hepatoma derived growth factor-like 1 \\
\hline Col6a1 & $3.10 \mathrm{E}-02$ & -2.71 & $12 / 16, p<0.05$ & collagen, type VI, alpha 1 \\
\hline Runx2 & $2.30 \mathrm{E}-02$ & -2.66 & $12 / 16, p<0.05$ & runt related transcription factor 2 \\
\hline Penk & $2.74 \mathrm{E}-02$ & -2.65 & $14 / 16, p=2.69 E-03$ & preproenkephalin \\
\hline Thy1 & $2.90 \mathrm{E}-02$ & -2.64 & $13 / 16, p=0.01$ & thymus cell antigen 1 , theta \\
\hline Pde1a & 4.73E-02 & -2.63 & $12 / 16, p<0.05$ & phosphodiesterase $1 \mathrm{~A}$, calmodulin-dependent \\
\hline Shank1 & 4.68E-02 & -2.57 & $12 / 16, p<0.05$ & SH3/ankyrin domain gene 1 \\
\hline Irak3 & 4.24E-02 & -2.54 & $11 / 16, p=0.13$ & interleukin-1 receptor-associated kinase 3 \\
\hline Olfr68 & $4.12 \mathrm{E}-02$ & -2.51 & $13 / 16, p=0.01$ & olfactory receptor 68 \\
\hline Krt5 & $2.26 \mathrm{E}-02$ & -2.41 & $13 / 16, p=0.01$ & keratin 5 \\
\hline Olfr355 & 2.83E-02 & -2.36 & $13 / 16, p=0.01$ & olfactory receptor 355 \\
\hline Ccdc113 & $2.21 \mathrm{E}-02$ & -2.32 & $13 / 16, p=0.01$ & coiled-coil domain containing 113 \\
\hline Thy1 & $3.18 \mathrm{E}-02$ & -2.31 & $11 / 16, p=0.13$ & thymus cell antigen 1, theta \\
\hline Wnt7a & 2.63E-02 & -2.26 & $12 / 16, p<0.05$ & wingless-related MMTV integration site 7A \\
\hline Slc9a4 & 4.83E-02 & -2.23 & $11 / 16, p=0.13$ & solute carrier family 9 (sodium/hydrogen exchanger), member 4 \\
\hline Adcy2 & $3.77 \mathrm{E}-02$ & -2.22 & $12 / 16, p<0.05$ & adenylate cyclase 2 \\
\hline Rgs6 & $1.56 \mathrm{E}-02$ & -2.19 & $13 / 16, p=0.01$ & regulator of G-protein signaling 6 \\
\hline Kera & $7.78 \mathrm{E}-03$ & -2.14 & $15 / 16, p=4.65 E-04$ & keratocan \\
\hline Mr1 & $5.31 \mathrm{E}-03$ & -2.14 & $15 / 16, p=4.65 E-04$ & major histocompatibility complex, class I-related \\
\hline Sp5 & 4.84E-03 & -2.13 & $12 / 16, p<0.05$ & trans-acting transcription factor 5 \\
\hline Apcdd1 & 7.13E-03 & -2.12 & $14 / 16, p=2.69 E-03$ & adenomatosis polyposis coli down-regulated 1 \\
\hline Osr2 & $1.87 \mathrm{E}-02$ & -2.08 & $13 / 16, p=0.01$ & odd-skipped related 2 (Drosophila) \\
\hline Lrrc17 & $2.83 \mathrm{E}-02$ & -2.05 & $11 / 16, p=0.13$ & leucine rich repeat containing 17 \\
\hline Pkp1 & $2.05 \mathrm{E}-02$ & -2.05 & $13 / 16, p=0.01$ & plakophilin 1 \\
\hline Klf4 & $4.08 \mathrm{E}-02$ & -2.03 & $11 / 16, p=0.13$ & Kruppel-like factor 4 (gut) \\
\hline C1qtnf7 & $9.12 \mathrm{E}-03$ & -2.02 & $13 / 16, p=0.01$ & $\mathrm{C} 1 \mathrm{q}$ and tumor necrosis factor related protein 7 \\
\hline $\mathrm{Ccl} 21 \mathrm{a}$ & 4.57E-02 & -2.00 & $11 / 16, p=0.13$ & chemokine (C-C motif) ligand 21A \\
\hline Kcnmb2 & $5.53 \mathrm{E}-03$ & -2.00 & $12 / 16, p<0.05$ & potassium large conductance calcium-activated channel, subfamily $\mathrm{M}$, beta member 2 \\
\hline
\end{tabular}


strain mice. ${ }^{[2]}$ This suggests that disruption of multiple genetic and environmental factors add to the increased penetrance of CLP seen in CL/Fr mice. ${ }^{[27-30]}$ Previous studies with $\mathrm{Pbx}$ mutants, $W n t 9 b-/-$, and mice with disrupted WNT signaling due to misexpressed genes such as sonic hedgehog $(S h b)$, showed reduced Trp63 expression in the facial prominence epithelium overlapping spatiotemporally with $W n t 9 b, W n t 3$, and other $W n t$ genes. ${ }^{[22,24]}$ Previous work suggested possible control and activation of Trp63 through canonical Wnts. From this, we hypothesized that decreased $W n t 9 b$ leads to reduced Trp63 in CL/Fr mice. However, our microarray data supported by qRT-PCR results did not indicate significant perturbations of $\operatorname{Trp} 63$ or Wnt3 in CL/Fr mice (Figure 1b). This suggests that Trp63 expression may be maintained in CL/Fr mice due to redundancy or compensation by other $W n t$ genes that are expressed within the facial prominences and that a dramatic decrease in expression of $W n t$ may be necessary for a reduction in $\operatorname{Trp} 63$ expression.

Regarding genes in the lf 2 locus, we identified two candidate genes from the array, Ube2ql1 and Adcy2, that may be affected in this region. In previous studies, it was postulated that gene(s) in the clf2 locus on chromosome 13, may contribute to CLP occurrence in "A" strain mice by interacting epistatically with $W n t 9 b$ and affecting the degree of methylation of the IAP transposon located 3' of $W n t 9 b$. Studies show that low methylation levels of the transposon is associated with CLP. ${ }^{[9,10,16]}$ Of the two genes detected in our microarray though, Ube2ql1 showed a greater statistical probability of being the gene affected in the clf2 locus (Table 3). Ube2ql1 is in the ubiquitin-conjugating enzyme family, and is responsible for catalyzing covalent attachment of ubiquitin to the target proteins in the second step of the ubiquitination reaction. The key player in the canonical $W n t$ program is $\beta$-catenin, a cytoplasmic protein that regulates a destruction complex that involves ubiquination for targeted proteasomal degradation. ${ }^{[31]}$ The brain and face share a close morphogenetic relationship and both genes are widely expressed in the brain during development. ${ }^{[32]}$ Thus, Ube $2 q 11$ and possibly $A d c y 2$ may be implicated indirectly in cleft development; however, no specific role in the epigenetic modification of IAP can be speculated based on our results and raw expression data from the array shows very low detection of transcripts. Thus, the identity of the direct mechanism by which clf2 modifies CLP in CL/Fr, remains elusive.

Additional genes that may contribute to the complicated genetic pathways of CLP were analyzed based on the microarray data, and we identified over 100 genes that are highly misexpressed ( $>2$-fold; p-value $\leq 0.05$ ). Many lie outside of the prospective critical loci. These genes may be involved in the CL/Fr mutation secondarily from pathways affected by the lf 1 or clf 2 mutations, may have independent roles in facial prominence morphogenesis, or be a product of feedback signaling resulting from abnormal molecular events occurring in cleft tissues. Evidence exists suggesting a number of these candidates direct morphogenetic processes such as cell proliferation, differentiation, and apoptosis. The runt-related transcription factor (Runx 2$)$ and odd-skipped related transcription factor $(\mathrm{Osr} 2)$ are two other genes associated with CLP that were significantly altered in our microarray analysis. Runx2 regulates the differentiation of mesenchymal stem cells into osteoblasts and bone formation. ${ }^{[33]}$ Most mice deficient in Runx 2 presented cleft palate. ${ }^{[34]}$ Osr2 is involved in regulating palatal shelf elevation and functions as a downstream target of Pax9 during palatogenesis. ${ }^{[35,36]}$ The downregulation of Runx2 (-2.66 fold; $\mathrm{p}<0.05)$ and $0 s r 2(-2.08$ fold; $\mathrm{p}<0.01)$ in $\mathrm{CL} / \mathrm{Fr}$ tissues may indicate a secondary effect resulting in cleft palate. Further investigation is necessary to confirm the expression values of these genes and to identify their role in craniofacial morphogenesis.

We were not able to clearly identify genes from our microarray analysis that $W n t 9 b$ is known to regulate. $W n t 9 b$ regulates FGF signaling pathways in the ectoderm of the nasal prominence. Reduced levels of Fgf8, 10 and 17 along with reduced cell proliferation of facial mesenchyme in mice deficient of $W n t 9 b$ has been reported. ${ }^{[23]}$ Furthermore, proliferation of the palatal mesenchyme was reduced in the palatal shelves of $\mathrm{CL} / \mathrm{Fr}$ mice. ${ }^{[37,38]}$ Our initial qRT-PCR studies on the most highly upregulated gene in the array, Sst (Table 1) showed a 1.72-fold up-regulationin CL/Fr tissues (Figure 5). Sst is a growth hormone-inhibiting hormone with many known functions, which include inhibiting cell proliferation and promoting apoptosis. ${ }^{[39]}$ Variable up-regulation of Sst in the facial prominences could lead to a decrease in growth hormone release, which may slow the growth of the MxPs and MNP and prevent fusion of the prominences in the transient nasal fin regions, leading to clefts in these individuals. Inhibition of cell proliferation by Sst is consistent with the effect seen in tissues with reduced FGF signaling as in $W n t 9 b$ knockout mice. It is possible that Sst may have even countered the effect of FGF signaling to inhibit cell proliferation in $\mathrm{CLP}(+)$ mice. However, further work will be required to understand this potential mechanism.

The $\mathrm{Ca}^{2+}$-calmodulin stimulated phosphodiesterase 1A (Pde1a), previously shown to regulate $\beta$-catenin/Tcf signaling was another highly misexpressed gene; downregulated 3.85 fold in the array (Table 3). Previous work demonstrated that inhibition of PDE1A in vascular smooth muscle cells inhibit $\beta$-catenin signaling through 
up-regulation of protein phosphatase 2A (PP2A), an isoform of which $(P p p 2 r 2 c)$ was up-regulated 3.02-fold in the array ${ }^{[40]}$ In addition to this, a gene encoding calciumbinding protein, calbindin-2 (Calb2), with roles in calcium homeostasis was also downregulated 3.64-fold in the array (Table 3). Thus, misregulation of these key players in the Pde1a/Pp2a/ $\beta$-catenin/Tcf signaling pathway may be contributing to the CLP defect. These genes and perhaps many of the other highly misregulated genes listed in Tables 2 and 3 may be targets of the $W n t / \beta$ catenin signaling or involved in unrelated pathways. This suggests that the etiology of CLP in CL/Fr is highly complex, and thus future studies should focus on elucidating the molecular mechanisms involved with these genes and the role they play in facial development.

\section{Conclusion}

The genetic mechanisms involved in the development of CLP are undoubtedly very complex. Wnt $9 b$ is shown here to be significantly downregulated in CL/Fr mice as seen in other "A" strain mice, emphasizing its important role in craniofacial morphogenesis. However, the higher penetrance of CLP in CL/Fr mice compared to other "A" strain mice suggests that other factors such as the misexpression of many other key genes outside of the prospective critical loci, and perhaps environmental factors as well, may play important roles in the development of CLP in CL/Fr. Future studies should focus on validating the highly misexpressed genes in the microarray. Subsequently, studies should be aimed at determining whether these genes are downstream targets of Wnt $9 \mathrm{~b}$ signaling, and then if so, identifying the mechanisms by which abnormal signaling by these genes leads to CLP.

\section{Acknowledgements}

Dr. Y. Lan provided the $W n t 9 b$ probe for $i n$ situ analysis. This work was supported by grants R01 DK064752 (SL), HCF 2001265 (SL), HCF 60316 (KSKF).

\section{References}

1. Prevalence (number of cases) of cleft lip and palate. National Institute of Dental and Craniofacial Research http://www.nidcr.nih.gov/ 2014 (accessed in Oct 25, 2016)

2. Parker SE, Mai CT, Canfield MA, Rickard R, Wang Y, Meyer R, Anderson P, Mason CA, Collins JS, Kirby RS, Correa A. Updated national birth prevalence estimates for selected birth defects in the United States. Birth Defects Res (Part A) 2010;88:1008-16.

3. Dixon MJ, Marazita ML, Beaty TH, Murray JC. Cleft lip and palate: synthesizing genetic and environmental influences. Nat Rev Genet 2011;12:167-78.

4. Kalter H. The history of the A family of inbred mice and the biology of its congenital malformations. Teratology 1979;20:213-32.
5. Juriloff, DM. Mapping studies in animal models. In: Wyszynski DF, editor. Cleft lip and palate. From origin to treatment. New York: Oxford Press; 2002. pp. 265-82.

6. Kadowaki S, Sakamoto M, Kamiishi H, Tanimura T. Embryologic features of term fetuses and newborns in CL/Fr mice with special reference to cyanosis. Cleft Palate Craniofac J 1997;34:211-7.

7. Juriloff DM, Harris MJ, Mah DG. The clf1 gene maps to a 2- to 3cM region of distal mouse chromosome 11. Mamm Genome 1996; 7:789.

8. JuriloffDM, Harris MJ, Brown CJ. Unraveling the complex genetics of cleft lip in the mouse model. Mamm Genome 2001;12:426-35.

9. Juriloff DM, Harris MJ, Dewell SL. A digenic cause of cleft lip in Astrain mice and definition of candidate genes for the two loci. Birth Defects Res A Clin Mol Teratol 2004;70:509-18.

10. Plamondon JA, Harris MJ, Mager DL, Gagnier L, Juriloff DM. The clf2 gene has an epigenetic role in the multifactorial etiology of cleft lip and palate in the $\mathrm{A} / \mathrm{WySn}_{\mathrm{S}}$ mouse strain. Birth Def Res A Clin Mol Teratol 2011;91:716-27.

11. Carinci F, Pezzetti F, Scapoli L, Martinelli M, Avantaggiato A, Carinci P, Padula E, Baciliero U, Gombos F, Laino G, Rullo R, Cenzi R, Carls F, Tognon M. Recent developments in orofacial cleft genetics. J Craniofac Surg 2003;14:130-43.

12. Carinci F, Scapoli L, Palmieri A, Zollino I, Pezzetti F. Human genetic factors in nonsyndromic cleft lip and palate: an update. Int J Pediatr Otorhinolaryngol 2007;71:1509-19.

13. Fontoura C, Silva RM, Granjeiro JM, Letra A. Association of WNT9B gene polymorphisms with nonsyndromic cleft lip with or without cleft palate in a Brazilian nuclear families. Cleft Palate Craniofac J 2015;52: 44-8.

14. He F, Chen Y. Wnt signaling in lip and palate development. Front Oral Biol 2012;16:81-90.

15. Juriloff DM, Harris MJ, McMahon AP, Carroll TJ, Lidral AC. Wnt9b is the mutated gene involved in multifactorial nonsyndromic cleft lip with or without cleft palate in $\mathrm{A} / \mathrm{WySn}$ mice, as confirmed by a genetic complementation test. Birth Defects Res A Clin Mol Teratol 2006;76:574-9.

16. Juriloff DM, Harris MJ, Mager DL, Gagnier L. Epigenetic mechanism causes Wnt9b deficiency and nonsyndromic cleft lip and palate in the $\mathrm{A} / \mathrm{Wy}_{\mathrm{S}} \mathrm{n}$ mouse strain. Birth Defects Res A Clin Mol Teratol 2014;100:772-88.

17. Livak KJ, Schmittgen TD. Analysis of relative gene expression data using real-time quantitative PCR and the 2(Delta DeltaC(T)) method. Methods 2001;25:402-8.

18. Bustin SA, Benes V, Garson JA, Hellemans J, Huggett J, Kubista M, Mueller R, Nolan T, Pfaffl MW, Shipley GL, Vandersompele J, Wittwer CT. The MIQE guidelines: minimum information for publication of quantitative real-time PCR experiments. Clin Chem 2009;55:611-22.

19. Lan Y, Ryan RC, Zhang Z, Bullard SA, Bush JO, Maltby KM, Lidral AC, Jiang R. Expression of $\mathrm{Wnt} 9 \mathrm{~b}$ and activation of canonical $\mathrm{Wnt}$ signaling during midfacial morphogenesis in mice. Dev Dyn 2006; 235:1448-54.

20. Fogelgren B, Kuroyama MC, McBratney-Owen B, Spence AA, Melahn LE, Anawati MK, Cabatbat C, Alarcon VB, Marikawa Y, Lozanoff S. Misexpression of Six2 is associated with heritable frontonasal dysplasia and renal hypoplasia in $3 \mathrm{H} 1 \mathrm{Br}$ mice. Dev Dyn 2008;237:1767-79.

21. Sokal RR, Rohlf FJ. Biometry: the principles and practice of statistics in biological research. New York (NY): WH Freeman; 1995. 
22. Ferretti E, Li B, Zewdu R, Wells V, Hebert J, Karner C, Anderson M, Williams T, Dixon J, Dixon M, Depew M, Selleri L. A conserved Pbx-Wnt-p63-Irf6 regulatory module controls face morphogenesis by promoting epithelial apoptosis. Dev Cell 2011;21: 627-41.

23. Jin Y-R, Han XH, Taketo MM, Yoon JK. Wnt9b-dependent FGF signaling is crucial for outgrowth of the nasal and maxillary processes during upper jaw and lip development. Development 2012;139: 1821-30.

24. Kurosaka H, Iulianella A, Williams T, Trainor P. Disrupting hedgehog and WNT signaling interactions promotes cleft lip pathogenesis. J Clin Invest 2014;124:1660-71.

25. Diewert VM, Wang K-Y, Tait B. A new threshold model for cleft lip in mice. Ann NY Acad Sci 1993;678:341-3.

26. Young N, Wat S, Diewert VM, Browder LW, Hallgrimsson B. Comparative morphometrics of embryonic facial morphogenesis: Implications for cleft-lip etiology. Anat Rec (Hoboken) 2007;290: 123-39.

27. Bronsky PT, Johnston MC, Sulik KK. Morphogenesis of hypoxiainduced cleft lip in CL/Fr mice. J Craniofac Genet Dev Biol Suppl 1986;2:113-28.

28. Martin DA, Nonaka K, Yanagita K, Nakata M. The effect of dam strain on the craniofacial morphogenesis of CL/Fr mouse fetuses. J Craniofac Genet Dev Biol 1995;15:117-24.

29. Nonaka K, Sasaki Y, Watanabe Y, Yanagita K, Nakata M. Effects of fetus weight, dam strain, dam weight, and litter size on the craniofacial morphogenesis of CL/Fr mouse fetuses affected with cleft lip and palate. Cleft Palate Craniofac J 1997;34:325-30.

30. Nonaka K, Sasaki Y, Martin DA, Nakata M. Effect of the dam strain on the spontaneous incidence of cleft lip and palate and intrauterine growth of CL/Fr mouse fetuses. J Assist Reprod Genet 1995;12:447-52.
31. Clevers H. Wnt/beta-catenin signaling in development and disease. Cell 2006;127:469-80.

32. Marcucio RS, Young NM, Hu D, Hallgrimsson B. Mechanisms that underlie co-varation of the brain and face. Genesis 2011;49: 177-89.

33. Ducy P, Zhang R, Geoffroy V, Ridall AL, Karsenty G. Osf2/Cbfa1: a transcriptional activator of osteoblast differentiation. Cell 1997;89: 747-54.

34. Aberg T, Cavender A., Gaikwad JS, Bronckers AL, Wang X, Waltimo-Siren J, Thesleff I, D'Souza RN. Phenotypic changes in dentition of Runx2 homozygote-null mutant mice. J Histochem Cytochem 2004;52:131-9.

35. Lan Y, Ovitt CE, Cho ES, Maltby KM, Wang Q, Jiang R. Oddskipped related $2(\mathrm{Osr} 2)$ encodes a key intrinsic regulator of secondary palate growth and morphogenesis. Development 2004;131: 3207-16.

36. Zhou J, Gao Y, Lan Y, Jia S, Jiang R. Pax9 regulates a molecular network involving Bmp4, Fgf10, Shh signaling and the Osr2 transcription factor to control palate morphogenesis. Development 2013;140: 4709-18.

37. Sasaki Y, Tanaka S, Hamachi T, Taya Y. Deficient cell proliferation in palatal shelf mesenchyme of CL/Fr mouse embryos. J Dent Res 2004;83:797-801.

38. Sasaki Y, Taya Y, Saito K, Fujita K, Aoba T, Fujiwara T. Molecular contribution to cleft palate production in cleft lip mice. Congenit Anom (Kyoto) 2014;54:94-9.

39. Barnett P. Somatostatin and somatostatin receptor physiology. Endocrine 2003;20:255-64.

40. Jeon K-I, Jono H, Miller C, Cai Y, Lim S, Liu X, Gao P, Abe J-I, Li J-D, Yan C. Ca2+/calmodulin-stimulated PDE1 regulates the betacatenin/TCF signaling through PP2A B56 gamma subunit in proliferating vascular smooth muscle cells. FEBS J 2010; 227:5026-39.

Correspondence to: Scott Lozanoff, PhD

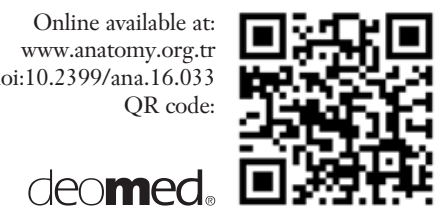

Department of Anatomy, Biochemistry and Physiology, University of Hawaii John A. Burns School of Medicine, Honolulu, Hawaii, USA

Phone: +1 8086921442

e-mail: lozanoff@hawaii.edu

Conflict of interest statement: No conflicts declared.

This is an open access article distributed under the terms of the Creative Commons Attribution-NonCommercial-NoDerivs 3.0 Unported (CC BY-NCND3.0) Licence (http://creativecommons.org/licenses/by-nc-nd/3.0/) which permits unrestricted noncommercial use, distribution, and reproduction in any medium, provided the original work is properly cited. Please cite this article as: Takagi B, Hong T, Hynd TE, Fong KSK, Fogelgren B, Nonaka K, Lozanoff S. Reduction in Wnt9b and associated gene expression in the embryonic midface of CL/Fr mice with heritable cleft lip and palate. Anatomy 2016;10(3):182-192. 